Kirstein, P; Lambrinos, L; (2007) Integrating Voice over IP Services in IPv4 and IPv6 Networks. In: Proceedings of the International Multi-Conference on Computing in the Global Information Technology. IEEE

\title{
ARTICLE
}

\section{Integrating Voice over IP services in IPv4 and IPv6 networks}

\author{
Lambros Lambrinos \\ Dept.of Communication and Internet studies \\ Cyprus University of Technology \\ Limassol 3603, Cyprus \\ lambros.lambrinos@cut.ac.cy \\ Peter Kirstein \\ Department of Computer Science \\ University College London (UCL) \\ Gower Street, London, WC1E 6BT, UK \\ p.kirstein@cs.ucl.ac.uk
}

\begin{abstract}
The transition from the current IPv4 network addressing model to the next generation IPv6 network addressing model has always been deemed as a lengthy process. As the deployment of IPv6 infrastructures evolves, interaction with the existing IPv4 networks is unavoidable. This interaction may occur at the network level (simple data pass-through) or at the application level (between IPv6 and IPv4 endpoints). In this paper we concentrate on application level interoperability and select an increasingly popular application: Voice Over IP (VoIP). The use of VolP technologies is becoming more widespread as end-users and network providers recognise the potential benefits that the usage of the application provides. It is important to provide an environment where IPv4 and IPv6 networks and applications / devices can interoperate in the context of VolP. This environment is based on an architecture that integrates all the components together (whether they support IPv4 or IPv6 or both) thereby providing ubiquitous access to IP telephony services based on the SIP signaling protocol. We present our experiences from realising the proposed architecture.
\end{abstract}

\section{Introduction}

The IPv6 network addressing protocol is considered the flagship protocol in the nextgeneration internet. The main benefits derived from the adoption of IPv6 include the large address space (128-bit compared with 32-bit in IPv4), better mobility support, easier security integration and flow labeling. The motivation behind the use of IPv6 and the expected benefits have already been extensively analysed and documented [1].

The transition process from IPv4 to IPv6 is underway but it is not entirely clear when it will start occurring on a large scale basis. This results in isolated islands of IPv6 networks with IPv4 in the backbone network. In the current Internet environment mechanisms such as Network Address Translation (NAT) [2] are used to reduce the need for public IPv4 addresses.

An application that will result in a high demand for public network addresses is Voice 
Kirstein, P; Lambrinos, L; (2007) Integrating Voice over IP Services in IPv4 and IPv6 Networks. In: Proceedings of the International Multi-Conference on Computing in the Global Information Technology. IEEE

over IP (VoIP). VolP is increasingly becoming very popular and major telecommunications equipment vendors are making related products. These new devices will attach to the network and hence require a public IP address so that they can be readily located. Moreover, wireless devices with VoIP capabilities are expected to become available soon so there will be more demand for public addresses as well as mobility support. These two factors will likely play an important role in speeding up IPv6 adoption.

The IP address shortage problem can be easily solved by providing all the new devices with IPv6 addresses. However, these new devices will not be able to communicate with the existing IPv4-based devices without some form of IPv4 to IPv6 translation. In an effort to utilise the best characteristics of the existing and future network technologies, we propose an architecture to integrate all the components together to provide ubiquitous access to IP telephony. Realising the architecture, we successfully built a testbed for VoIP interaction between multiple users behind both IPv4 and IPv6 networks. In order to implement the architecture a variety of components were utilized; our emphasis was on open-source software paying particular attention (as expected) to the existence of IPv6 support.

The paper is organized as follows: a background introduction to IPv6 and the advantages it offers is followed by a description of the relevant VoIP protocols. The overall architecture which integrates IPv4 and IPv6 networks along with the Public Services Telephone Network (PSTN) is then presented. The various components (SIP proxy, PSTN gateway, IPv6-IPv4 gateway, user agents and IP phones) are described in detail along with the rationale behind our decision to utilise them. We then present our experiences from realizing the network architecture by illustrating the various interoperability tests that were carried out on the infrastructure and describing the results and problems we identified. The paper ends with our conclusions and plans for future work.

\section{Voice over IP technologies}

In today's fast-pace environment, the Internet offers a multitude of applications which users can utilise in order to communicate with each other. These applications range from the very simple such as email and text-based chat, to the more advanced such as instant messaging and videoconferencing. In the last few years, telecommunications operators make use of IP technologies during traditional voice calls in order to carry the voice data for some segments of a call. Businesses are also beginning to realise the potential benefits that can be offered when voice is carried over the packet-switched IP infrastructure. The most important advantages offered are the savings in call costs and the requirement for a single integrated network rather than two independent voice and data networks.

Initial implementations of VoIP technology were initially based on proprietary protocols. Due to the enormous research and commercial interest in the technology, proprietary technologies have gradually given way to more open interoperability standards such as H.323 [3], Megaco [4] and MGCP [5]. Until recently, the H.323 protocol from the ITU-T was the most widely adopted. H.323 offers a complete solution for call setup and control but it may be inflexible in some scenarios. The Session Initiation Protocol (SIP) [6] from the IETF follows a more flexible approach allowing many potential applications. While 
Kirstein, P; Lambrinos, L; (2007) Integrating Voice over IP Services in IPv4 and IPv6 Networks. In: Proceedings of the International Multi-Conference on Computing in the Global Information Technology. IEEE

there is still a large number of deployments based on H.323, SIP is establishing itself as the signaling protocol for current and future multimedia communications and its use in the context of VolP is the focus of this paper.

\subsection{The Session Initiation protocol}

The Session Initiation Protocol (SIP) is an application-layer signaling protocol that is used to setup and terminate multimedia sessions such as VoIP calls. SIP is currently used in a variety of applications including instant messaging and multi-way videoconferencing. SIP communication is text-based and consists of requests and responses. Hence, a request contains information about its origin (and in some cases the details of the SIP entities it has traversed before reaching its destination) so that the response can be routed back.

Sip uses Uniform Resource Identifiers (URIs) [7] to depict the user's "identification" which is considered unique. A SIP URI consists of the username, the domain the user is registered with and potentially a port number e.g.sip:joe.bloggs@mysip.site.com. This "identity" essentially follows the user; this is a highly useful feature as users are contactable with the same URI irrespective of their location, a feature that can assist in mobility scenarios. Of equal importance, is the fact that the callee's location is totally transparent to the caller.

During the session setup process, session descriptions [8] are exchanged between the interacting parties in order to agree on a set of parameters for the session with the most important one being the media encoding types. More details on SIP and the basic dialogues for establishing connections are given in [9].

\subsubsection{SIP servers}

In its simplest form, SIP can operate under a peer-to-peer model where a user sends a direct invite to another user in order to setup a call. As the protocol was becoming more widely adopted, both in respect of the categories of applications it was utilized in (e.g. VolP, instant messaging etc.) and the number of users, SIP servers became a vital part of the support infrastructure. There are three different of categories of SIP servers:

- registrar - holds location information and preferences for the users registered with the server. Calls destined for a user are forwarded to the user's current location (i.e. IP address) where they will be handled by the User Agent

- proxy - the major point of contact for request routing. Requests are either forwarded directly to the callee (if the callee's current location is known) or to another server that may have further information on the callee's location

- redirect - call requests received are replied with information about the next server to be contacted in order to locate the callee

A single host usually performs the role of all the above but in some scenarios it may be desirable to have separate hosts for load balancing and security purposes.

\subsubsection{SIP User Agents}


Kirstein, P; Lambrinos, L; (2007) Integrating Voice over IP Services in IPv4 and IPv6 Networks. In: Proceedings of the International Multi-Conference on Computing in the Global Information Technology. IEEE

SIP user agents (UAs) are software programs or hardware devices that use SIP for the setup and disconnection of a call. Software-based UAs are very widely used as the equipment required (microphone and headset) is readily available. Many UAs are freely available either as open source packages (e.g. rat[10]) or as commercial releases without the most advanced functionality (e.g. XLite [11]). As expected, the functionality, the implementation quality and the performance during calls varies between the various UAs.

End-user hardware devices for VolP come in two flavours: Analogue Telephone Adapters (ATAs) that allow users to plug in their normal home telephone and IP phones which are telephone sets with network connectivity. Nowadays there are many manufacturers of SIP-compliant phones with varying features. Some IP phones also provide video transmit/receive capabilities.

As mentioned earlier, a user agent has to register with a SIP registrar to notify the registrar of the user's credentials and current network location. This information will be used during call setup where the mapping of the username to the actual IP address of the callee is handled by the proxy and is of no concern to the caller.

\subsection{The Real-time Transport Protocol}

It is important to note that SIP does not provide for session information and status after the session has been established. For the transport of the audio data between sender and receiver, the Real-time Transport Protocol (RTP) [12] is used in combination with the Real-time Transport Control Protocol (RTCP) [13] that carries information on session statistics. Audio generated from the microphone is encoded according to the coding algorithm agreed during the setup of the session before it is encapsulated in a RTP packet and sent to the network. At regular intervals, senders and receivers use the RTCP protocol to transmit statistical information (e.g. packet loss and delay) regarding the data sent / received.

\section{An architecture for providing VolP over IPv6 networks}

The aim of our work was to provide an operating environment for VolP that allows calls between IPv4 and IPv6 user agents regardless of the heterogeneity in the network addressing protocols used.

The main components of the system are presented in Figure 1. The SIP server (SER) acts as SIP registrar and proxy for setting up calls. The IPv4-IPv6 gateway acts as the translating bridge between the two networks. The PSTN gateway provides access to the PSTN so that users on normal analogue telephones can interact with users using the VOIP infrastructure. Finally, the MCU acts as a conferencing application for multiparty communication. The architecture also includes the various user agents. Each component is discussed in detail below. 
Kirstein, P; Lambrinos, L; (2007) Integrating Voice over IP Services in IPv4 and IPv6 Networks. In: Proceedings of the International Multi-Conference on Computing in the Global Information

Technology. IEEE

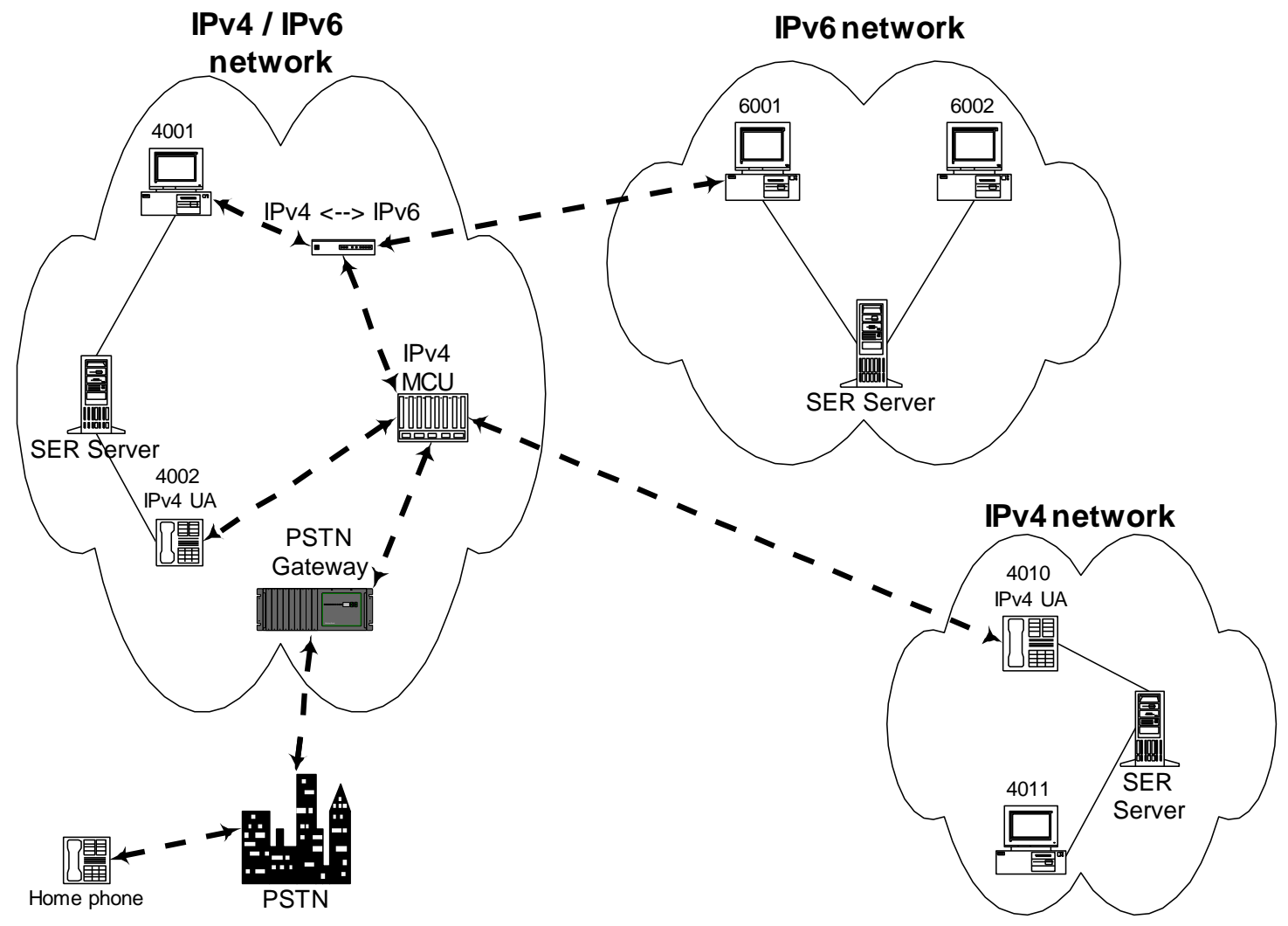

Figure 1 A VolP architecture for IPv6

\subsection{SIP Express Route}

The SIP Express Router (SER) platform [14] is the heart of the system's architecture. It includes functionality that allows it to act as a SIP registrar, proxy and redirect server. SER is available as open source software and it is a very well designed system that provides high performance as it is capable of handling user registrations in the order of thousands. Our reason for choosing it over other alternatives was its stability, scalability and proven functionality. More importantly, SER incorporates IPv6 support thereby allowing IPv6 user agents to utilise its services.

Through additional modules, SER also includes support for other functions such as instant messaging, NAT traversal, RTP relaying, and accounting, authorization and authentication (AAA) services.

\subsection{The SIP IPv4 - IPv6 protocol Gateway}

The SIP protocol gateway (SIP-PGW) acts as a translator between IPv4 and IPv6 networks. As shown in Figure 2, SIP-PGW consists of two components: the mini-SIPproxy (msp) and the UDP-forwarding (ufwdd) deamon. The msp performs the necessary modifications on SIP messages as these flow between the IPv4 and IPv6-based UAs so that these can be correctly interpreted at their destination. The two components communicate using UDP in order to allocate addresses and ports. The ufwdd then performs the forwarding of the actual media data between the participating UAs. 
Kirstein, P; Lambrinos, L; (2007) Integrating Voice over IP Services in IPv4 and IPv6 Networks. In: Proceedings of the International Multi-Conference on Computing in the Global Information Technology. IEEE

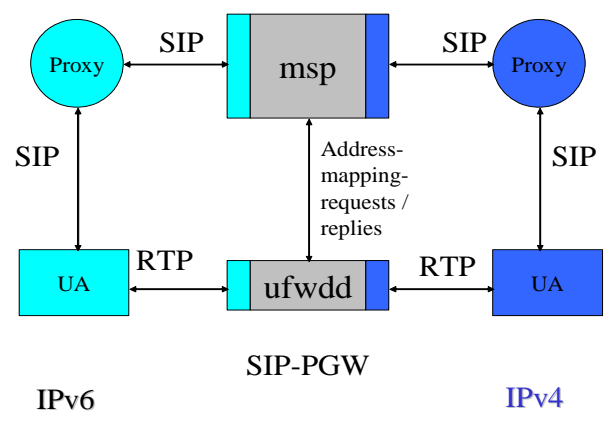

Figure 2: The SIP Protocol Gateway (SIP-PGW) architecture

It is important to note that SIP-PGW operates in conjunction with two SIP proxies (one in IPv4 and one in IPv6) for request routing. More information on the operation and modifications performed by the SIP-PGW can be found in [15].

\subsection{PSTN Gateway}

The Public Switched Telephone Network (PSTN) gateway links the system with the PSTN network through ISDN lines. Using the gateway, user agents can communicate with analog telephones connected to the PSTN. Moreover, a person can dial in the gateway and select a specific extension or even connect to an on-going SIP conferencing session.

\subsection{Asterisk}

Asterisk [16] is a software system that provides advanced PBX functionality. It offers VoIP support using multiple protocols (SIP, H.323 and IAX) and also allows seamless interconnection with the PSTN. Although Asterisk is only IPV4 compliant, it can facilitate multiparty conferencing for simultaneous interaction between multiple participants. This is achieved through the MeetMe application which takes the role of an MCU and is part of the Asterisk platform.

\subsection{User Agents}

A number of user agents were selected for the interoperability tests. The softphones used were X-Lite [17] a very well developed and freely available commercial software and kphone [18] that was based on open source code and included IPv6 support. For hardware IP phones, the Cisco 7960 was used. Although, X-Lite and the Cisco IP phone did not have IPv6 support built-in, they were highly suitable for the IPv4 to IPv6 interconnection tests.

\section{Deployment and testing}

To ascertain the feasibility of the proposed architecture a testbed was deployed and a number of interoperability tests were executed.

\subsection{Testbed implementation}


Kirstein, P; Lambrinos, L; (2007) Integrating Voice over IP Services in IPv4 and IPv6 Networks. In: Proceedings of the International Multi-Conference on Computing in the Global Information Technology. IEEE

The components of the architecture proposed in Figure 1 were deployed at different sites as part of the 6NET IST project [19] during which a high-speed native IPv6 network was in operation. SER servers were installed in both IPV4 and IPv6 environments providing registrar and proxy services to the respective UAs. The SIP-PGW was running on a dual-stack machine at the "borderline" between the different environments. As required, each SER configuration "pointed" to the respective part of the SIP-PGW to allow communication between the heterogeneous environments.

The user agents were installed in multiple locations. In order to have consistency in the numbering and user accounts a numbering plan was devised. Two ranges of numbers were assigned to users; 4xxx and 6xxx covering the IPv4 and IPv6 UAs respectively. A further range 8xxx was allocated for multi-way conference calls. Moreover, calls destined for the PSTN had to start with the digit ' 9 ' which was removed before the call was dialled.

\subsection{Advanced Communication scenarios}

A number of interoperability tests were carried out in order to assess the functionality of the various components when used in combination with each other. We paid particular attention to the testing between user agents located at heterogeneous addressing networks and multiparty conferencing.

Tests between components in the same address family were also carried out. For each other. For IPv6, one-to-one calls between two instances of an IPv6 softphone (kphone) IPv4, softphones and the Cisco IP phones had no problems communicating with were also successful. In this instance, the IPv6 address of the callee's SIP proxy is obtained (using an AAAA DNS request) and the request is forwarded by the caller's proxy.

\subsubsection{IPv4 to IPv6}

The most important aspect of the implementation was the provision of seamless communication between IPv4 and IPv6 user agents. Under this scenario, we successfully tested calls between an IPv6 softphone and an IPv4 Cisco IP phone and between an IPv6 softphone and an Ipv4 softphone. Calls were successful when initiated by either of the parties involved.

As an illustrative example, Figure 3 depicts the exchange of messages between the various entities when an IP phone places a call to an IPv6 softphone. For clarity, the SIP proxies are omitted as we assume that where applicable they just forward the messages to the user agents concerned. The call was made to: sip:6002@sipserver.cs.ucl.ac.uk. As the dialed extension number started with the digit ' 6 ' the server forwarded the SIP message to the IPv6-based SIP server via the SIP-PGW. The IPV6 SIP server then looked up its database for the location of the user 6002 and forwarded the SIP INVITE. Based on record-route information in the SIP messages, replies from the IPV6 UA are routed back to the IP phone via the SIP-PGW.

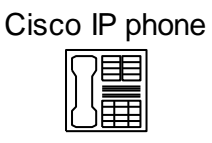

124.16.67.21
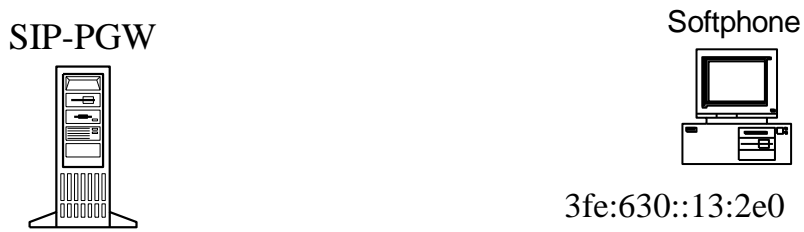

3fe:630::13:2e0

124.16.55.16

abe:2a0::123:a2:e3 
Kirstein, P; Lambrinos, L; (2007) Integrating Voice over IP Services in IPv4 and IPv6 Networks. In: Proceedings of the International Multi-Conference on Computing in the Global Information Technology. IEEE

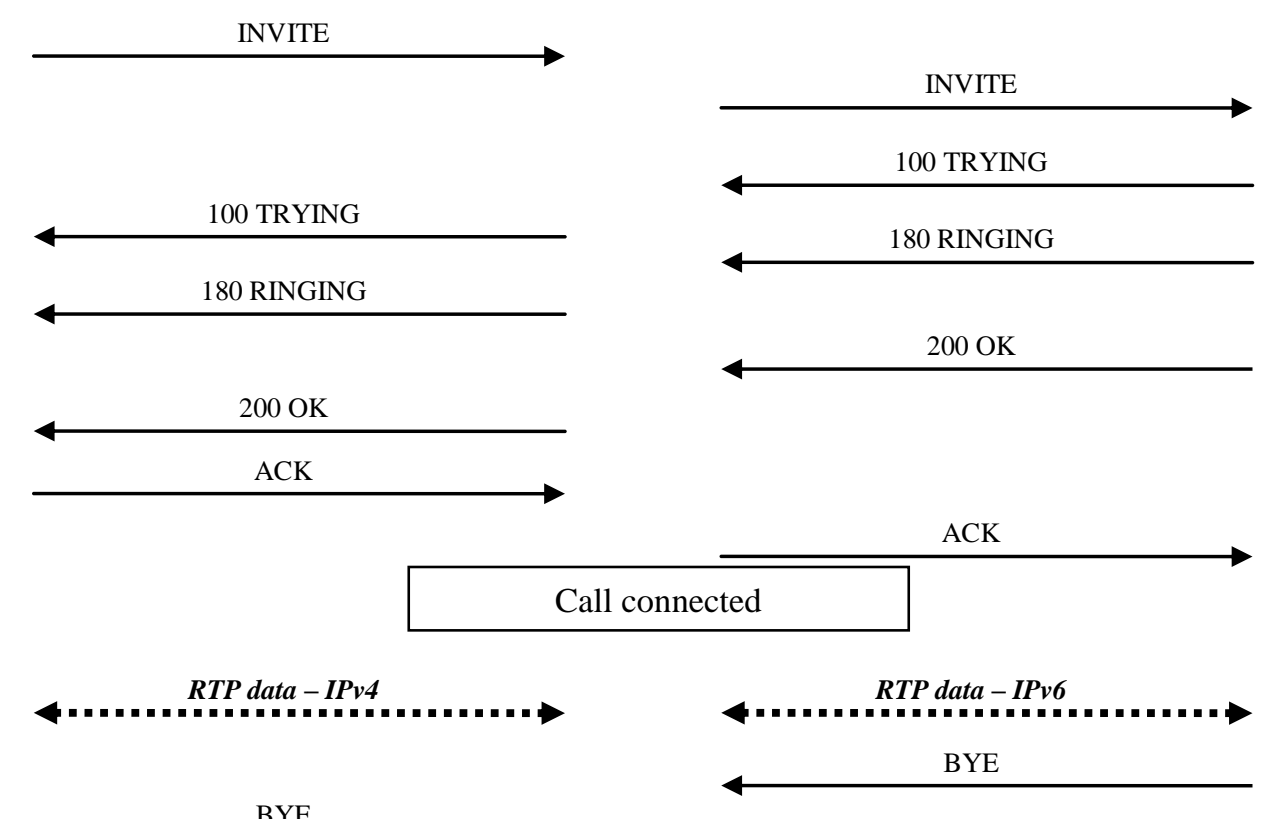

Figure 3. IPv4 - IPv6 VolP session setup

Once the call is established, the actual data flows using the RTP/RTCP protocols and the SIP-PGW (ufwdd deamon) perform the address mapping for the data packets. When the session is to be terminated, the party wishing to do that sends a SIP BYE message to the other party using the same way that SIP messages for call setup were sent.

\subsubsection{IPv6 to PSTN}

Originally, access to the PSTN from an IPv6 UA has been problematic. This seems to be due to codec support issues and SIP functionality in the PSTN gateway. As an alternative solution the use of the SEMS ISDNGW software [20] in conjunction with an AVM Fritz ISDN card was considered. This operated successfully under Suse Linux.

\subsubsection{Multi-party conferencing}

Another test scenario involved multiparty conferencing between an IPv6 softphone, an IPv4 softphone and an IPv4 Cisco phone. The MCU receives the streams and multiplexes them before transmitting the combined stream to the various members of the conference. As the MCU is only IPv4 capable, where there are participants using an IPv6 agent, the combined stream is sent to the IPv4 interface of the SIP-PGW for forwarding. This example is illustrated in Figure 1, the dashed arrows depict the data flows between the various participants and intermediary entities.

\subsection{Component evaluation}


Kirstein, P; Lambrinos, L; (2007) Integrating Voice over IP Services in IPv4 and IPv6 Networks. In: Proceedings of the International Multi-Conference on Computing in the Global Information

Technology. IEEE

As mentioned earlier, a number of components were used in the implementation of our architecture and during the interoperability tests. Generally, there were only minor problems observed with the overall implementation.

SER was quite stable and no problems have been identified with regards to its operation as a SIP registrar and proxy. The Ipv4/6 gatewaying functionality in SIP-PGW is correct and the Asterisk MCU handled the data translated from the IPv6 UAs without any problems.

With regards to the user agents, the performance of Kphone as an IPv6-capable softphone was not adequate in a number of instances. This has largely been the case with calls over the WAN where poor quality audio was experienced. The most likely reason is kphone's lack of packet loss and delay compensation algorithms. Both the Cisco IP phone and X-Lite software performed adequately during IPv4 and IPv6 calls.

\section{Conclusion}

VolP is deemed as one of the driving forces behind the adoption of IPv6. In this paper we propose an architecture that integrates a collection of components together to provide ubiquitous access to VolP telephony for both IPv4 and IPv6 user agents. It is imperative to have an intermediate environment as it is expected that parts of the Internet will remain with IPv4 for some time to come. Our realization of the architecture we proposed has resulted in a fully functional VolP system based entirely on SIP.

We expect that softphone developers and IP phone manufacturers will soon start incorporating IPv6 functionality in their products. In the meantime, we shall try and improve our solution by identifying and incorporating any new components that may have become available. Moreover, we will try to provide more integrated access to PBX facilities and examine how to provide enhanced support for mobile agents. 